\title{
Understanding the Characteristics of Oleuropein for Table Olive Processing
}

\author{
Yasin Ozdemir ${ }^{*}$, Engin Guven and Aysun Ozturk \\ Ataturk Central Horticultural Research Institute, Food Technology Department, Yalova, Turkey \\ *Corresponding author: Yasin Ozdemir, Ataturk Central Horticultural Research Institute, Food Technology Department, Yalova, Turkey, Tel: 0090 226 81425; Fax: \\ 0090226814 1146; E-mail: yozdemir@yalovabahce.gov.tr
}

Rec date: Apr 29, 2014, Acc date: May 13, 2014, Pub date: May 29, 2014

Copyright: $\odot 2014$ Ozdemir Y, et al. This is an open-access article distributed under the terms of the Creative Commons Attribution License, which permits unrestricted use, distribution, and reproduction in any medium, provided the original author and source are credited.

\begin{abstract}
Phenolic compounds make up 2-3\% (w/w) of olive flesh, whose most abundant part is oleuropein which is responsible for the characteristic bitter taste of olive fruits. Oleuropein is a heterosidic ester of elenolic acid and hydroxytyrosol and possesses beneficial effects on human health. But most fundamental and important step of table olive processing is to reduce level of oleuropein because olive fruits can only be consumed after de-bittering procedures. Oleuropein accumulates during fruit growth and is slowly converted to elenolic acid glucoside and demethyloleuropein as the fruit ripens. Harvest maturation affects the table olive processing factors, according to change on oleuropein content of olive fruits. More severe applications are required for processing of olive which has high oleuropein content. Also oleuropein and its hydrolysis end products (elenolic acid and aglycone) inhibit the activity of a number of microorganisms involved in the lactic fermentation of olives. Oleuropein was significantly affected by both acid and base treatment, resulted in the liberation of hydroxytyrosol. Bitter taste of olive fruit can be eliminated by removing the oleuropein out of the olive or chemical or enzymatic hydrolysis of oleuropein in table olive processes. Understanding the characteristics of oleuropein for table olive processing helps manufacturer to produce high quality table olives and researchers to develop innovative methods for table olive production. So that this research was aimed to explore the characteristics of oleuropein and its effects on table olive processes.
\end{abstract}

Keywords: Oleuropein, olive; Table olive; Bitterness; Table olive processing

\section{Introduction}

Oleuropein, a bitter-tasting secoiridoids glycoside present in olive leaves and fruits, is the main phenolic compound in olive fruits although it undergoes a notable decrease during the course of fruit ripening and processing. For this reason the nutritional intake of oleuropein is usually considered as questionable $[1,2]$. Although the majority of the polyphenols found in table olives are derivatives of its hydrolysis, in most cases, the concentration of oleuropein in edible sources like table olives and olive oil has been found to be very low [3]. Olive fruit can only be consumed after debittering procedures which consist of removal or degradation of oleuropein [4].

The main purpose of table olives processing is the removal of bitterness related to oleuropein [5]. For this purpose, several processes mainly based on alkaline hydrolysis or diffusion in brine is employed to lead to oleuropein decrease and hydroxytyrosol increase [6]. Also bitter taste of olive fruit can be eliminated by enzymatic hydrolysis of oleuropein or semi drying processing of olives in table olive processes $[6,7]$. Understanding the characteristics of oleuropein for table olive processing helps manufacturer to produce high quality table olives and researchers to develop innovative methods for table olive production. This review was aimed to explore the characteristics of oleuropein, remove or degradation of oleuropein and transformation of oleuropein during olive processing.

\section{Characteristics of Oleuropein}

Oleuropein is an important constituent of olive fruits and a widely studied natural product with numerous health related activities.
Phenolic compounds make up 2-3\% (w/w) of olive flesh, whose most abundant part is oleuropein which is responsible for the characteristic bitter taste of olive fruits [8]. Oleuropein was discovered in 1908 by Bourquelot and Vintilesco, and its structure was specified as being that of a heterosidic ester of elenolic acid and dihydroxyphenylethanol [9]. Oleuropein belongs to a very specific group of coumarin-like compounds, called secoiridoids, which are abundant in Oleaceas, Gentianales, Cornales and many other plants [10]. Oleuropein is water-soluble component which has a potential to use as a food supplement [8]. Olive fruit is rich in the secoiridoids oleuropein, demethyloleuropein, ligstroside, and in their hydrolytic derivatives. Figure 1 shows the chemical structure of oleuropein which possesses antioxidant and radical scavenging properties. Antioxidant activity of oleuropein is mainly due to the hydroxytyrosol moiety in its structure [11]. Also oleuropein contributes to the prevention of atherosclerosis by inhibiting the oxidation of LDL and by scavenging several species that react with oxygen in the vascular wall $[12,13]$.

Oleuropein preparations have been claimed for several pharmacological effects. Despite these potential health benefits, oleuropein is removed from olives due to its bitter taste $[14,15]$. Oleuropein and its derivatives are inhibitors of lactic acid bacteria growth which has important role in table olive fermentation [16]. Many researchers have demonstrated this compound has antimicrobial property, so that it might be used as an alternative food additive [4] (Figure 1).

The change in oleuropein content was reflected by a corresponding change in total phenols. Oleuropein content is changed between increased from $20-150 \mathrm{mg} \mathrm{g}^{-1}$ (expressed as $\mathrm{mg}$ equivalents phenol per gram dry mass) during maturation [17]. Elenolic acid glucoside and demethyloleuropein are glycosylated derivatives of oleuropein which accumulate during olive (Olea europaea) maturation.These 
compounds appear simultaneously with a fall in oleuropein content and an increase in esterase activity [18]. Olives when green are in their most bitter state because of the high levels of phenolic compounds, oleuropein in particular. After the olive fruit reaches the black-ripe stage it loses moisture and the bitter oleuropein breaks down to the point where some varieties are edible without requiring any processing [8]. Olive fruit maturation stage, process type and type of fermentation highly influence the level of polyphenolic constituents and especially oleuropein [19]. During table olive processing, the levels of oleuropein are markedly reduced and hence the fruit is debittered [20]. Oleuropein content of unprocessed olive has been under investigation for many years [21]. Despite the great importance of oleuropein from the health point of view, commercial market table olives have not been equally studied [22].

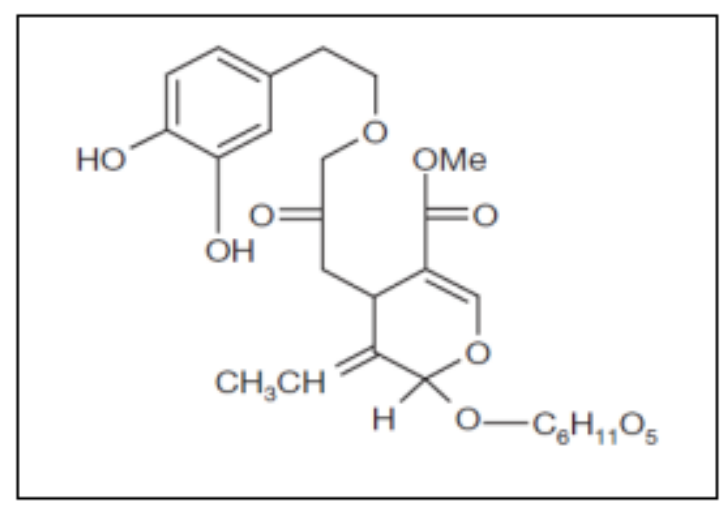

Figure 1: Chemical structure of oleuropein

\section{Removal of Oleuropein}

Oleuropein is extracted from the raw flesh by diffusion when olives are soaked in water or brine during processing. Oleuropein is leached out of the olives and removed from the tank when the soaking solution is discarded. During brining, water-soluble oleuropein and other phenolic compounds, sugars, vitamins and minerals leach out of the olive flesh by this way bitterness of the olives is resolved $[6,20]$. Figure 2 shows salt, oleuropein and water soluble component transport between whole olive and brine. When olives are subject to soaking and/or natural fermentation without prior lye treatment, oleuropein is diluted and/or partially destroyed so the final products retain some level of bitterness [8].

When olive is soaked in brine, fermentation takes place during the exchange of material between the olive and brine. Salt moves from saline to the olive and oleuropein and water soluble components move from olive to the saline. This diffusion between the brine and olive flesh takes place normally due to osmotic pressure difference [23].

Slitting, cracking, bruising or destining the olives facilitates extraction of oleuropein by reducing the barrier effect of olive skin and shorten debittering time [8]. Slitting or cracking mostly used for natural green olive processing in brine $[6,8]$.

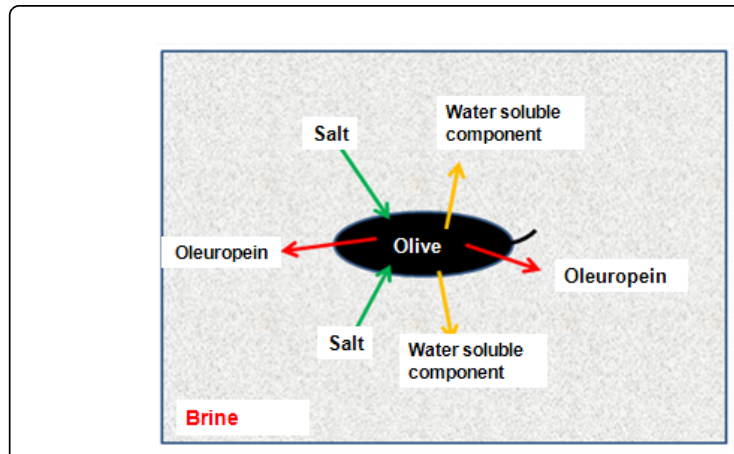

Figure 2: Component transport between olive and brine [23].

In Spanish or Turkish traditional olive processing method which consists of spontaneous fermentation in brine some weight (stone or rock mostly used for this purpose) is placed on the olives, therefore, a forced diffusion takes place between brine and olive flesh. Figure 3 shows the component transport between olive and brine under forced diffusion. This applied pressure accelerates the debittering, causes decrease in water content of final product and an increase on the firmness of olive tissue [23].

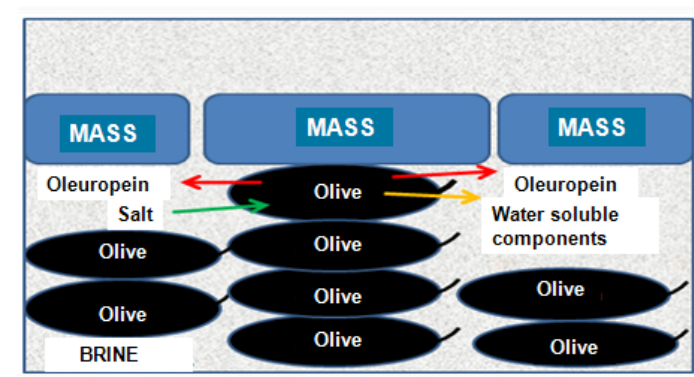

Figure 3: Component transport between olive and brine under forced diffusion [23].

\section{Degradation of Oleuropein}

Oleuropein possesses beneficial effects on human health. But most fundamental and important step of table olive processing is to reduce level of oleuropein because olive fruits can only be consumed after debittering procedures [8]. Debittering occurs by the hydrolysis or breakdown, of oleuropein into less bitter by-products, possibly by the action of lye, some microorganisms or enzymes. Small amounts of sugar are released during the hydrolysis of oleuropein which also contribute to fermentation [6]. The procedure for preparing Spanishstyle green olives consists of treating the fruits with dilute $\mathrm{NaOH}$ solution to hydrolyze their natural bitterness (oleuropein), followed by one or two water washes to remove the excess alkali [24]. Figure 4 shows the scheme for the transformation of oleuropein and its hydrolysis products during Spanish-style green olive processing. However, the sodium hydroxide causes many chemical and physical changes in the fruit, leading to the loss of soluble constituents and nutrients responsible for the typical properties of the best quality table olives [25]. 


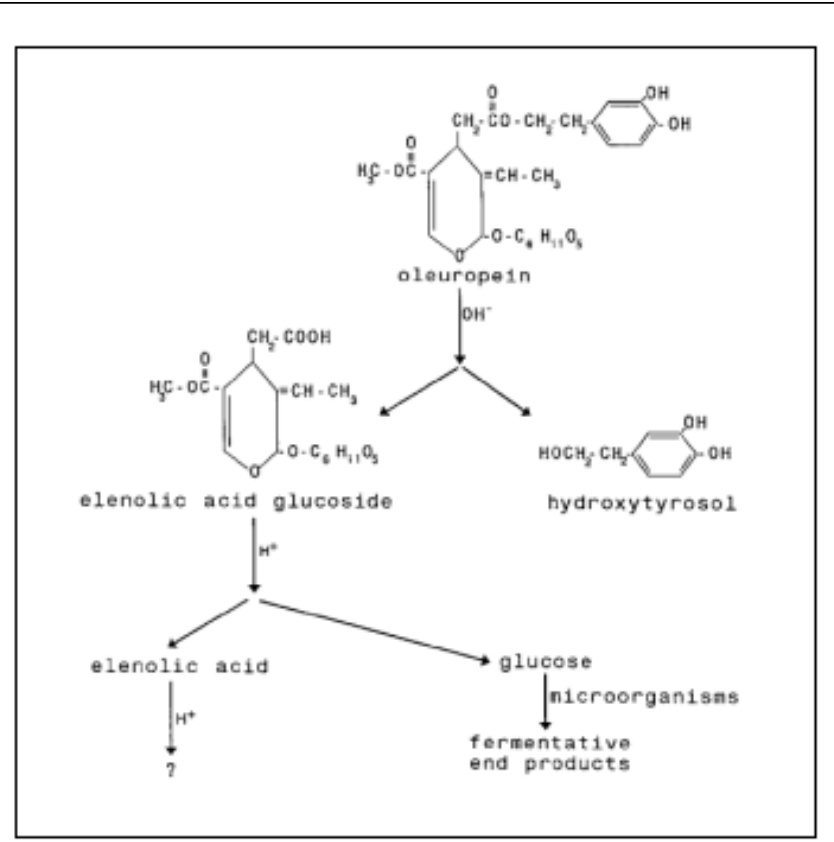

Figure 4: Scheme for the transformation of oleuropein and its hydrolysis products during Spanish-style green olive processing $[24]$.

Oleuropein was significantly affected by both acid and base treatment, resulted in the liberation of hydroxytyrosol. Oleuropein is degraded by lye but not in the acid environments created by fermentation. Recent research has identified some strains of fermentative lactic acid bacteria that can breakdown oleuropein through enzymatic reactions, hence debittering the olives as well as participating in the fermentation process. This biotechnology could replace the use of lye in table olive processing [8].

Enzymatic hydrolysis of the glycosidic linkage of oleuropein by $\beta$ glucosidase activity was considered as an alternative processing method to lye and/ or brine treatment. $\beta$-glucosidase activity results in formation of glucose and oleuropein aglycone [7].

Figure 5 shows the scheme of enzymatic degradation of oleuropein and formed products during degradation. The glycosidases are important group of enzymes for many biochemicals, biomedical and industrial applications and catalyze the hydrolysis of the glycosidic bonds. There are some investigations on microbial origin $\beta$ glucosidases for substituting $\mathrm{NaOH}$ treatment in table olive processing $[7,26]$.

Several groups have investigated the in vitro degradation of oleuropein by different microorganisms. Recently, it has been reported that Lactobacillus plantarum strains isolated from olive fermentation brines can hydrolyse oleuropein in vitro by means of $\beta$-glucosidase. Some strains of $L$. plantarum can hydrolyse not only oleuropein but also the aglycone derivative $[28,29]$.

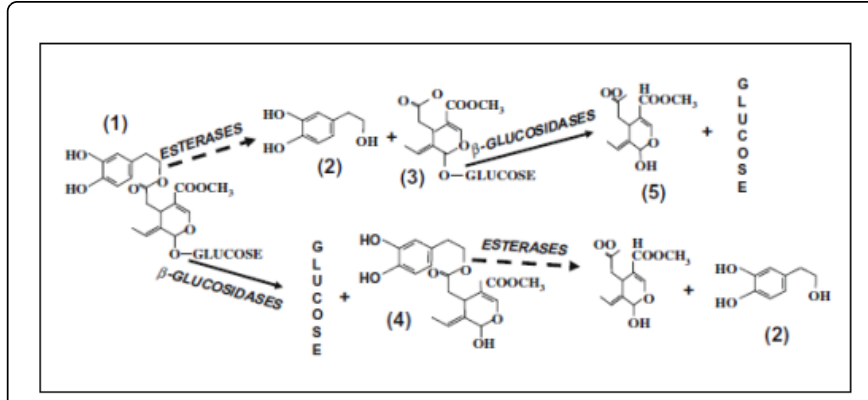

Figure 5: Scheme for enzymatic degradation of oleuropein and products formed according to the reaction type: (1) oleuropein, (2) hydroxytyrosol, (3) glucosyl derivate, (4) aglycone, and (5) elenolic acid [27].

Enzymatic hydrolysis of oleuropein, particularly by $\beta$-glucosidase, is well established in the literature for both bacteria and fungi [30]. This kind of enzymatic activity is important in olive production when deriving from lactic acid bacteria, while fungal growth may negatively affect both the nutritive and aesthetic value of the product with the presence of mycelium on the surface and mycotoxin production $[29,31]$.

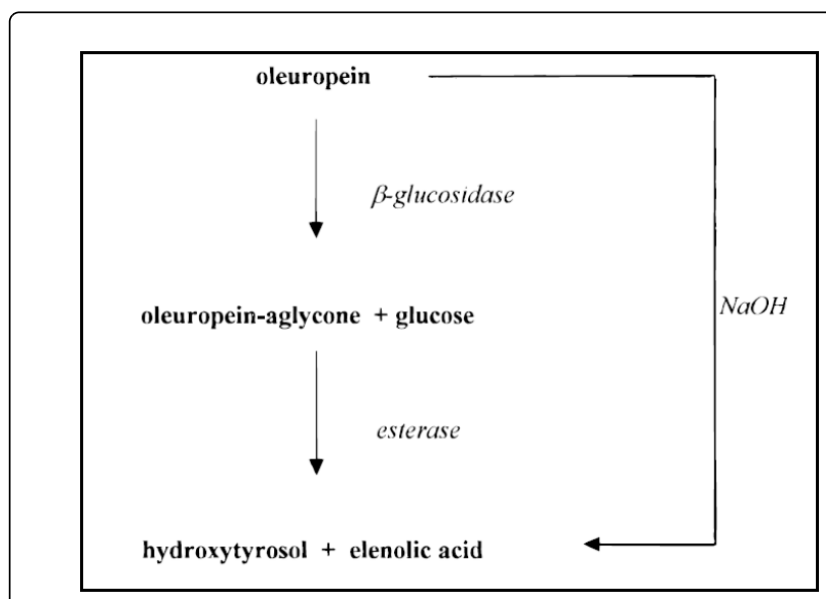

Figure 6: Proposed pathway for oleuropein metabolism [30].

A proposed degradative pathway for oleuropein degradation is shown in Figure 6. Briefly, the proposed pathway involves a two-step process: the first step is the hydrolysis of the glycosidic linkage of the oleuropein by b-glucosidase with formation of oleuropein-aglycone, the first observable intermediate in the process, in a second step the amount of aglycone formed is hydrolyzed to elenolic acid and hydroxytyrosol probably by esterase activity $[27,32]$.

Dehydration of olive fruit by means of heat (in oven or under sun) in the Mediterranean area is used as a traditional approach for degradation of oleuropein. This latter technology provides olives ready for consumption within 1-3 days [6,33]. Hot air dehydration of blanched olives (dips in boiling water for a few minutes) followed by a 2 or 3 days storage in salt is used in Italy for the Majatica di Ferradina cultivar [33]. 
Dry salt debittering is one of the oldest methods which consist of placing the olive fruits in disposed layers with dry salt [23,34]. In olive debittering during the dry-salt process, due to the enzymatic oxidation of oleuropein in the olive flesh resulting in no leaching of the phenolic glucoside in the generated brine. The osmotic pressure provoked by the salt on the olives gave rise to the rupture of the tissues thereby putting polyphenols into contact with polyphenoloxidase [34].

\section{Transformation of Oleuropein during Table Olive Processing}

Harvest maturation affects table olive processing factors, according to change on oleuropein content of olive fruits. More severe applications are required for processing of olive which has high oleuropein content. Also oleuropein and its hydrolysis end products (elenolic acid and aglycone) inhibit the activity of a number of microorganisms involved in the lactic fermentation of olives [8].

There are several processing methods to obtain palatable olives. In natural olive processing (no lye treated) requires a brining stage during which the olives are fermented to have the characteristic texture, aroma and flavour. In naturally processed olives, hydrolysis of oleuropein and other glycosides is slow and can even occur after the olives are packed, resulting in secondary fermentation and stability problems [24]. However, black olives can be debittered before brining by successive treatments with dilute $\mathrm{NaOH}$ solution, used mainly for green olives [35]. The lye treatment and subsequent washing process comprises a very complex mechanism of removal of some water soluble compounds, like reducing sugar and organic acids, from the olive fruit as well as oleuropein [6].

Various studies have indicated that during the $\mathrm{NaOH}$ step in Spanish-style green olive processing, hydroxytyrosol (3,4dihydroxyphenylethanol) is formed [36]. Later studies have indicated that the other part of the oleuropein moiety is not modified by the alkali, although the concentration of elenolic acid glucoside diminished during the fermentation period. This glucoside is also known as oleoside 11-methyl ester [24,37,38]. Alkaline treatment does not allow the cleavage of the oleuropein moiety into its three components: hydroxytyrosol, glucose and elenolic acid, only the ester bond between hydroxytyrosol and elenolic acid glucoside is cleaved [38]. During the fermentation step of Spanish-style green olives, there is an acid hydrolysis of elenolic acid glucoside to glucose and elenolic acid, as shown in Figure 4. Elenolic acid is comparatively unstable and tends to degrade due to the acid conditions by the medium [6]. The glucose formed is utilized as substrate by the microorganisms present in the fermentation brines of Spanish-style green olives [6,24].

Oleuropein is hydrolyzed by oleuropeinolytic L. plantarum strains [39]. Process kinetics indicates that the bacterial strains initially hydrolyze the oleuropein by means of $\beta$-glucosidase action with formation of an aglycone (the first observable intermediate compound). This derivative gives rise to 2-(3,4-dihydroxyphenyl) ethanol and elenolic acid by esterase action $[25,39]$.

Oleuropein can be hydrolyzed by using $\beta$-glucosidases into glucose and oleuropein aglicone which further bio transforms to other nonbitter tasting metabolites. The use of $\beta$-glucosidase prevents the loss of fermentable material during the washing step of lye treatment, enhances the initial growth of lactic acid bacteria and results in a favored end-product with high sensory quality $[7,29,39]$.

\section{Discussion}

The fundamental and most important step of table olive processing is debittering. During debittering processes, the levels of oleuropein are markedly reduced either by leaching or hydrolysis, hence the olive is debittered $[6,20]$. Keeping in brine and treatment with $\mathrm{NaOH}$ are used two main methods in table olive industry. However, these two methods have disadvantages. These disadvantages of brine debittering need a long time (6-8 months) and the final product has high salt content (\%5-7). The disadvantages of debittering by $\mathrm{NaOH}$ are degradation of other phenolic compounds with oleuropein, nutrient losses caused by washing for removing the excess $\mathrm{NaOH}$ from olive and the formation of high amounts of waste water. For this reason, there is a need to develop new debittering methods to produce table olive. New technologies are needed in order to reduce the length of the debittering process and to reduce or complete replace the use of $\mathrm{NaOH}$ and the subsequent neutralizing washes or brine debittering processes. As oleuropein is water-soluble, it is extracted from the raw flesh by diffusion when olives are soaked in water or brine during processing. Slitting, cracking or bruising the olives facilitates extraction of oleuropein. However, important to processing, oleuropein is degraded by lye but not in the acid environments created by fermentation $[8,40]$.

Despite extensive studies on the optimization of different table olive processing techniques, there are only several studies on developing of new processing techniques $[8,9]$. Extensive studies should be carry on biodegradation of oleuropein by lactic acid bacteria species associated with the natural fermentation process. Lactic starters should also be determined and selected according to their potential for biological debittering and to improving the sensorial characteristics of fermented olives $[9,39]$.

In search for alternative processing methods to lye and/or brine treatment, enzymatic hydrolysis of the glycosidic linkage of oleuropein by $\beta$-glucosidase activity could be taken into consideration. The $\beta$ glucosidase activity on oleuropein results in formation of glucose and oleuropein aglycone [41,42]. The glycosidases catalyze the hydrolysis of the glycosidic bonds and are an important group of enzymes for many biochemicals, biomedical and industrial applications. $\beta$ glucosidases of microbial origin were investigated for substituting $\mathrm{NaOH}$ treatment in olive processing [26,29].

The biodegradation of oleuropein to hydroxytyrosol and other simple phenolic compounds by $L$. plantarum, involving $\beta$-glucosidase and esterase enzymes was reported $[28,43,44]$. According to tolerance to olive leaf extract (up to $10 \%, \mathrm{w} / \mathrm{v}$ ) and oleuropein $(1 \%, \mathrm{w} / \mathrm{v}), L$. plantarum, $L$. pentosus and $P$. pentosaceus can be suggested to use as starters in the controlled fermentation of non debittered green olives [31,39]. L. plantarum showed the highest percent of strains producing $\beta$-glucosidase and degrading oleuropein as the sole carbon source, followed by L. pentosus, P. pentosaceus and L. brevis [39].

Olive growers and table olive processors have a strong interest in using natural processing methods such as brine fermentation and salt/ heat drying methods rather than processing with lye in worldwide $[6,8]$. So that researchers who are interested in table olive processes should be focus on developing new debittering systems and technologies to reduce the production cost and waste material and improve the final product quality. This review presents the characteristics, removing and degradation of oleuropein and transformation of oleuropein during table olive processes. This will 
also help to researcher who is willing to develop innovative table olive production techniques.

\section{Acknowledgement}

This review was prepared within the scope of "Table Olive Production with Drying Process and Product Characterization" project which was founded by General Directorate of Agricultural Researches and Policies, Food, Agriculture and Livestock Ministry of Turkey.

\section{References}

1. Malik NSA, Bradford JM (2006) Changes in oleuropein levels during differentiation and development of floral buds in 'Arbequina' olives. Sci Horticult 110: 274-278.

2. Ferreira D, Guyot S, Marnet N, Delgadillo I, Renard MG, et al. (2002) Composition of phenolic compounds in Portuguese pear (Pyrus communis L. Var. S. Bartolomeu) and changes after sun drying. J Agr Food Chem 50: 4537-4544.

3. Ben Othman N, Roblain D, Thonart P, Hamdi M (2008) Tunisian table olive phenolic compounds and their antioxidant capacity. J Food Sci 73: 235-240.

4. Yildiz G, Uylaser V (2011) Doğal bir antimikrobiyel: oleuropein. Journal of Agricultural Faculty of Uludag University 25: 131-142.

5. Bianchi G (2003) Lipids and phenols in table olives. Eur J Lipid Sci Technol 105: 229-242.

6. Garrido Fernandez A, Fernandez Diez M J, Adams MR (1997) Table olives. Chapman Hall Press.

7. Tuna S, Akpinar-Bayizit A (2009) The use of $\beta$-glucosidase enzyme in black table olives fermentation. Not Bot Hort Agrobot 37: 182-189.

8. Kailis SG, Harris D (2007) Producing Table Olives. Landlinks Press.

9. Panizzi L, Scarpati ML, Oriente G (1960) Chemical structure of oleuropein, bitter glucoside of olive with hypotensive activity. Gazz Chim It al 90: 1449-1485.

10. Soler-Rivas C, Espin JC, Wichers HJ (2000) Oleuropein and related compounds. J Sci Food Agr 80: 1013-1023.

11. Benavente GO, Castilloa J, Lorentea J, Ortuñob A, Del Riob JA (2000) Antioxidant activity of phenolics extracted from Olea europaea L. leaves. Food Chemistry 68: 457-462.

12. Coni E, Di Benedetto R, Di Pasquale M, Masella R, Modesti D, et al. (2000) Protective effect of oleuropein, an olive oil biophenol, on low density lipoprotein oxidizability in rabbits. Lipids 35: 45-54.

13. Visioli F, Bellomo G, Galli C (1998) Free radical scavenging properties of olive oil polyphenols. Biochem Biophys Res Commun 247: 60-64.

14. Syed HO (2010) Oleuropein in olive and its pharmacological effects. Scientia Pharmaceutica 78:133-154.

15. Sudjana AN, Carla DO, Vanessa R, Nooshin R, Justin N et al. (2009) Antimicrobial activity of commercial Olea europaea (olive) leaf extract". International Journal of Antimicrobial Agents 33: 461-463.

16. Barba RJL, Balbuena BM, Jimenez DR, Garcia GP, Fernandez GA (1993) Inhibition of Lactobacillus plantarum by polyphenols extracted from two different kinds of olive brine. J Appl Bacteriol 74: 15-19.

17. Ryan D, Robards K, Lavee S (1999) Changes in phenolic content of olive during maturation. International Journal of Food Science \& Technology 34: $265-274$

18. Amiot MJ, Fleuriet A, Macheix JJ (1989) Accumulation of oleuropein derivatives during olive maturation. Phytochemistry 28: 67-69.

19. Romero C, Brenes M, Yousfi K, Garcia P, Garcia A, et al. (2004) Effect of cultivar and processing method on the contents of polyphenols in table olives. J Agric Food Chem 52: 479-484.

20. Kailis SG, Harris D (2004) Establish protocols and guidelines for table olive processing in Australia. Rural Industries Research and Development Corporation Report.
21. Amiot MJ, Fleuriet A, Macheix JJ (1986) Importance and evolution of phenolic compounds during growth and maturation. J Agric Food Chem 34 823-826.

22. Boskou G, Salta FN, Chrysostomou S, Mylona A, Chiou A, et al. (2006) Antioxidant capacity and phenolic profile of table olives from the Greek market. Food Chem 94: 558-564.

23. Ozdemir Y, Akcay ME, Kurultay S (2011) Olive debittering methods and physical and chemical changes during debittering. Proceedings of National Olive Congress.

24. Brenes M, de Castro A (1998) Transformation of oleuropein and its hydrolysis products during Spanish-style green olive processing. J Sci Food Agric 77: 353-358.

25. Marsilio V, Lanza B, de Angelis M (1996) Olive cell wall components: physical and biochemical changes during processing. J Sci Food Agr 70: 35-43.

26. Briante R, La Cara F, Febbraio F, Barone R, Piccialli G, et al. (2000) Hydrolysis of oleuropein by recombinant $\beta$-glycosidase from hyperthermophilic archaeon Sulfolobus solfataricus immobilised on chitosan matrix. J Biotechnol 77: 275-286.

27. Segovia-Bravo KA, Jarén-Galán M, García-García P, Garrido-Fernández A (2009) Browning reactions in olives: mechanism and polyphenols involved. Food Chemistry 114: 1380-1385.

28. Marsilio V, Lanza B, Pozzi N (1996) Progress in table olive debittering: degradation in vitro of oleuropein and its derivatives by Lactobacillus plantarum. JAOCS 73: 593-597.

29. Ciafardini G, Zullo BA (2000) $\beta$-glucosidase activity in olive brine during the microbiological debittering process. Adv Food Sci 22: 69-76.

30. Panagou EZ (2006) Greek dry-salted olives: Monitoring the dry-salting process and subsequent physicochemical and microbiological profile during storage under different packing condition at 4 and $20^{\circ} \mathrm{C}$. LWT Food Sci Technol 39: 322-329.

31. Ciafardini G, Marsilio V, Lanza B, Pozzi N (1994) Hydrolysis of oleuropein by Lactobacillus plantarum strains associated with olive fermentation. Appl Environ Microbiol 60: 4142-4147.

32. Marsilio V, Lanza B (1998) Characterisation of an oleuropein degrading strain of Lactobacillus plantarum combined effects of compounds present in olive fermenting brines (phenols, glucose and nacl) on bacterial activity transformation of oleuropein during olive processing. J Sci Food Agric 76: 520-524.

33. Gambella F, Piga A, Agabbio M, Vacca V, D'hallewin G (2000) Effect of different pretreatments on drying of green table olives (Ascolana tenera var.). Grasas y Aceites 51: 173-176.

34. Ramírez E, García-García P, de Castro A, Romero C, Brenes M (2013) Debittering of black dry-salted olives. European Journal of Lipid Science and Technology 115: 1319-1324.

35. Leal-Sanchez MV, Ruiz-Barba JL, Sanchez AH, Rejano L, Jimenez-Diaz R, et al. (2003) Fermentation profile and optimization of green olive fermentation using Lactobacillus plantarum LPCO10 as a starter culture. Food Microbial 20: 421-430.

36. Amiot MJ, Tachini M, Fleuriet A, Macheix JJ (1990) The technological debittering process of olives: characterization of fruits before and during alkaline treatment. Sci Aliments 10: 619-631.

37. Damtoft S, Franzyk H, Jensen SR (1995) Biosynthesis of iridoids in Syringa and Fraxinus secoiridoid precursors. Phytochemistry 40: 773-784.

38. Brenes M, Rejano L, Garcia P, Sanchez AH, Garrido A (1995) Biochemical changes in phenolic compounds during Spanish-style green olive processing. J Agric Food Chem 43: 2702-2706.

39. Ghabbour N, Lamzira Z, Thonart P, Cidalia P, Markaouid M, et al. (2011) Selection of oleuropein-degrading lactic acid bacteria strains isolated from fermenting Moroccan green olives. Grasas y Aceites 62: 84-89.

40. Savas E, Uylaser V (2013) Quality improvement of green table olive cv. 'Domat' (Olea europaea L.) grown in Turkey using different de-bittering methods. Not Bot Horti Agrobo 41: 269-275. 
Citation: Ozdemir Y, Guven E, Ozturk A (2014) Understanding the Characteristics of Oleuropein for Table Olive Processing. J Food Process Technol 5: 328. doi:10.4172/2157-7110.1000328

Page 6 of 6

41. Materassi R, Miclaus N, Pelagatti O (1975) Hydrolysis of oleuropein in yeasts. Ann dell Inst Speriment per ka Elaiotecn 5: 53-65.

42. Limiroli R, Consonni R, Ottolina G, Marsilio V, Bianchi G, et al. (1995) $1 \mathrm{H}$ and 13C NMR characterisation of new oleuropein aglycones. J Chem Soc Perkin Transac 1: 1519-1523.

43. Landete JM, Curiel JA, Rodriguez H, de las Rivas B, Munoz R (2008) Study of the inhibitory activity of phenolic compounds found in olive products and their degradation by Lactobacillus plantarum strains. Food Chem 107: 320-326.

44. Rodriguez H, Curiel JA, Landete JM, de las Rivas B, de Felipe FP, et al. (2009) Food phenolics and lactic acid bacteria. Int J Food Microbiol 132: 79-90. 